\title{
On the Usability of Electroencephalographic Signals for Biometric Recognition: A Survey
}

\author{
Su Yang and Farzin Deravi, Member, IEEE
}

\begin{abstract}
Research on using electroencephalographic signals for biometric recognition has made considerable progress and is attracting growing attention in recent years. However, the usability aspects of the proposed biometric systems in the literatures have not received significant attention. In this paper, we present a comprehensive survey to examine the development and current status of various aspects of electroencephalography (EEG)-based biometric recognition. We first compare the characteristics of different stimuli that have been used for evoking biometric information bearing EEG signals. This is followed by a survey of the reported features and classifiers employed for EEG biometric recognition. To highlight the usability challenges of using EEG for biometric recognition in real-life scenarios, we propose a novel usability assessment framework which combines a number of user-related factors to evaluate the reported systems. The evaluation scores indicate a pattern of increasing usability, particularly in recent years, of EEG-based biometric systems as efforts have been made to improve the performance of such systems in realistic application scenarios. We also propose how this framework may be extended to take into account Aging effects as more performance data becomes available.
\end{abstract}

Index Terms-Biometrics, electroencephalography (EEG), feature classification, feature extraction, usability.

\section{INTRODUCTION}

$\mathbf{E}$ LECTROENCEPHALOGRAPHY (EEG) is the (generally noninvasive) recording of the electrical activities of the brain along the scalp, measuring the small voltage fluctuations resulting from ionic currents within the brain [1]. One of the earliest investigations of brain-related functions was the monitoring the electroencephalographic signals of animals (rabbits' and monkeys' brains, in 1890) [2], [3]. The first human EEG was recorded in 1924 [4], later the EEG gradually became a useful tool in diagnosing brain disease (epileptic seizures [5], for instance). Considering the physiological differences between the brains of different people, EEG signals may be expected to possess the potential to not only indicate the brain's functions, but also dissimilarities between individuals as manifested by the electrical activity of their brains. Such an assumption has led to

Manuscript received September 30, 2015; revised September 27, 2016; accepted February 20, 2017. Date of publication April 12, 2017; date of current version November 13, 2017. This work was supported in part by the University of Kent academic on-line database service. This paper was recommended by Associate Editor J. del R. Millan. (Corresponding author: Su Yang.)

The authors are with the School of Engineering and Digital Arts, University of Kent, Canterbury, CT2 7NZ, U.K. (e-mail: sy91@kentforlife.net; F.Deravi@kent.ac.uk).

Color versions of one or more of the figures in this paper are available online at http://ieeexplore.ieee.org.

Digital Object Identifier 10.1109/THMS.2017.2682115 attempts at developing EEG-based person recognition systems, where EEG signals are used as a biometric modality.

The EEG signal has been employed for biometric purposes by a number of researchers in laboratory environments (for example in [6]); however, many problems still remain and need to be addressed before considering its application in real-life scenarios.

Previous surveys in [7] and [8] have addressed the theoretical aspects of EEG-biometric systems, reviewing the state-of-theart methods and their future perspectives. The goal of this investigation is to review the recent work on EEG-based biometric recognition systems while focusing on issues of practicality and usability which are key to achieving the wider deployment of such systems. This study is organized into four major sections: A review of the sensors and stimuli used to trigger and acquire EEG signals for person recognition is presented in Section II. In Section III, a survey of the techniques used for generating features from EEG signals provided. Section IV describes the relevant techniques for feature classification. Section V first explores a series of factors that affect the usability of an EEG biometric system, followed by introducing a novel metric which combines these factors to provide a better measurement of the usability and practicality of such systems. This section also contains suggestions for how this usability assessment framework may be extended to take into account Aging effects as more performance data becomes available. Section VI presents the overall conclusions and suggestions for further work.

\section{Signal ACQUisition}

Two types of sensor system have been used for biometric purposes: 1) medical-grade sensor systems and 2) low-cost sensor systems. The medical-grade systems conventionally contain a large number of electrodes for data capture; the sensors usually need to be moistening by electrolytes (e.g., saline solution). Such a sensor system generally produces high quality signals, but the deployment of the wet sensors could be impractical in EEG biometric applications. The distribution of the electrodes over the scalp conventionally follows the 10-20 system [9] or 10-10 system [10] (see Fig. 1). The 10-20 system and 10-10 system are internationally recognized methods to describe and apply the location of scalp electrodes in the context of an EEG test or experiment [11]. On the other hand, low-cost sensor systems typically contain a small number of electrodes (down to a single electrode), using dry sensor(s) to reduce the cost and improve the usability of the system. Usually only the frequency components below $50 \mathrm{~Hz}$ are taken into 


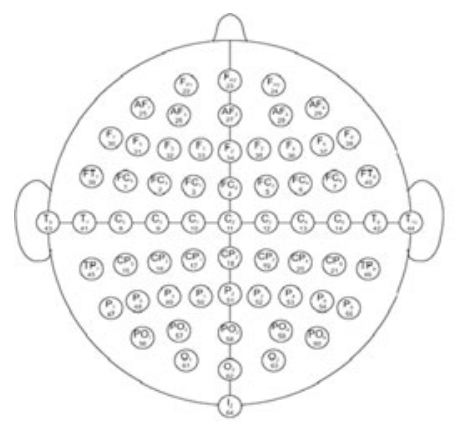

(a)

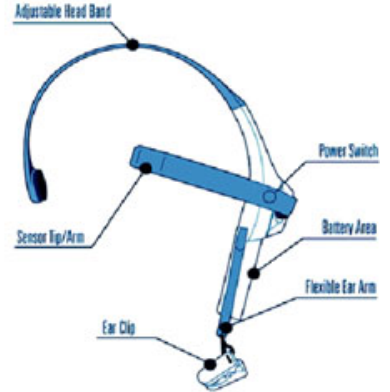

(b)
Fig. 1. (a) 64 electrodes positioning based on 10-10 system [10] and (b) single Fp1 electrode low-cost headset [13].

TABLE I

List of MARKet AVAILABLE Low-Cost EEG HeAdSETS

\begin{tabular}{lccc}
\hline \hline Device & Electrode & Sensor & Released \\
\hline NeuroSky MindSet [17] & 1 & dry & 2007 \\
Neural Impulse Actuator [18] & 3 & dry & 2008 \\
Emotiv EPOC [16] & 14 & wet & 2009 \\
Mindflex (Uses NeuroSky chips) & 1 & dry & 2009 \\
MindWave [17] & 1 & dry & 2011 \\
XWave headset (NeuroSky chips) & 1 & dry & 2011 \\
Melon Headband [19] & 4 & dry & 2014 \\
HiBrain [20] & 1 & dry & 2014 \\
iFocusBand [21] & 1 & dry & 2014 \\
Muse [22] & 4 & dry & 2014 \\
OpenBCI [23] & 8 or 16 & dry/wet & 2014 \\
Aurora Dream Headband [24] & 1 & dry & 2015 \\
Emotiv Insight [16] & 5 & dry & 2015 \\
\hline \hline
\end{tabular}

consideration in EEG biometric recognition. The typical EEG frequency bands considered for analysis are named: Delta $(<4$ $\mathrm{Hz})$, theta $(4-7 \mathrm{~Hz})$, alpha $(8-15 \mathrm{~Hz})$, beta $(16-31 \mathrm{~Hz})$, and gamma ( $>32 \mathrm{~Hz})[12]$.

EEG is a relatively new biometric modality, many sensor systems used in the research for EEG person recognition are directly those used in the medical field. Therefore, the number of electrodes usually is large and the sensors are scattered all over the scalp [9]. Typically, an EEG data acquisition software system, such as the BCI2000, is used to facilitate data collection [14], [15]. On the other hand, to be considered as a successful biometric modality, good recognition performance is necessary but may not be enough: The ease of deployment and low cost of a sensor system may also be similarly important in practical applications. Researchers have, therefore, been testing the performance of low-cost sensor headsets for biometric recognition. Popular headsets such as Emotiv [16] and Neurosky products [13] have been tested in biometric scenarios. Emotiv EPOC is the first low-cost sensor system using wet electrodes for signal acquisition [16].

Table I presents some commercial low-cost EEG sensor systems, which have or potentially could be used for biometric person recognition.

An essential consideration in EEG data acquisition for biometric person recognition is the state of the brain and the nature of its activity. The identity-information bearing signals that are produced by the brain may crucially depend on the type of mental task in which the user is engaged. Often there may need to be a predefined stimulus to evoke the desired brain activity for producing consistent results. The choice of stimulus that would result in optimal production of identity-bearing signals for biometric person recognition purposes is currently a subject of research. In the following sections, some relevant published approaches are surveyed covering three types of brain stimulation: Resting state, sensory (audio/visual) stimuli, and cognitive tasks-(verbal instructions).

\section{A. Resting State}

For the data acquisition process during the resting state, usually the subject is asked to sit on a chair in a quiet environment, either with eyes open or closed. The resting state scheme is the least demanding in terms of the need for additional equipment to generate an external stimulus; the users passively produce EEG signals without needing to follow additional instructions during the data collection.

La Rocca et al. [25] reported person identification performance using two resting state subsets of a relatively large (108 subjects) publicly available EEG database [10]: One with eyes open and the other with eyes closed. Of the one minute's EEG recording per subject, data of $10 \mathrm{~s}$ were used for testing and the rest for training. Using conventional power spectral features and a functional connectivity feature that they proposed, performance of $100 \%$ CRR was achieved by the fusion between sensors at the match score level, for each feature type separately. Fraschini et al. [26] employed the same dataset for a verification scenario, an equal error rate (EER) of $4.4 \%$ was obtained using the "eigenvector centrality" features extracted from gamma band $(30-50 \mathrm{~Hz})$.

A potential obstacle to using the resting state EEG data for biometrics recognition may be the ambiguity of the instruction "to rest" for users, which may be interpreted and acted on by even the same subject at different times in different ways, resulting in incommensurable data. By ensuring that the instruction is as clear as possible and that the subject undertakes a consistent mental activity at each session, it is likely that more distinctive biometric information would be produced.

\section{B. Sensory Stimuli}

An event-related potential (ERP) is the measured brain response that is the direct result of a specific sensory, cognitive, or motor event [27]. The P300 (P3) wave is an ERP component [28] which can be detected as a waveform between 250 and $500 \mathrm{~ms}$ after a visual stimulus is presented to the user. Researchers have used this particular waveform for person recognition.

Palaniappan et al. [29] employed the P300 components for feature extraction using an EEG cap with 61 electrodes while 10 subjects viewed a set of standardized pictures originally proposed in [30]. The maximum identification rate achieved was 95\% using the data recorded in a single session (part of the session used for training and the rest for testing). Similar but improved approaches have been tested using databases with larger population leading to maximum peak recognition rate of $98.12 \%$ with a database of 102 subjects [31]-[35].

One possible drawback of using visual stimuli for biometric applications is the need for external devices to produce the 
stimulus and generate the required EEG signals. This may result in biometric systems that are complex and costly compared with alternatives based on using the resting state or directed cognitive activity.

\section{Intentional Cognitive Activities}

Given the above-mentioned limitations of the resting state and sensory stimulus approaches for EEG biometric applications, researchers have explored other approaches which may result in brain signals that are more repeatable, and potentially more individually distinctive. A cognitive stimulus involves directing the subject(s) to perform various intentional tasks during the data collection: This could be either actual physical movement tasks or imagery tasks, where a particular physical movement is only imagined. The use of cognitive stimuli was first reported in 2005 when EEG data recorded during the performance of a variety of mental tasks (including: Mathematical calculation, geometric figure rotation, and mental letter composition) were first used in an identification scenario to distinguish between four subjects [36].

Marcel et al. [37] used the data captured while subjects imagining hand movements for a biometric authentication scenario. Power Spectral Density (PSD) features of EEG signals from different subjects were compared using Gaussian mixture models and, a half total error rate of $7.1 \%$ was reported for nine subjects using eight electrodes (using 16 min of the recorded data for training and $4 \mathrm{~min}$ for testing).

Chuang et al. [38] reported a system which employed only a single Fp1 electrode (NeuroSky MindSet); Two data acquisition sessions were conducted on separate days, each session lasted for 40-50 min. Different activities were performed, including: Object Counting, etc. The half total error rate of $1.1 \%$ for a database of 15 subjects was reported. However, an identification rate of only $22 \%$ was achieved using the same database.

Similar to the EEG signals captured during the resting state, the mental/cognitive task-stimuli also suffer from the ambiguity problem. However, usually the challenge of employing such tasks not only stems from the ambiguity of the task instruction and its interpretation, but also in the identification of the taskrelated segments of the overall signal that needs to be used for data analysis. Although there are established signal processing techniques and statistical techniques to extract these correlates of different mental tasks in the recorded brain activity when sensory stimuli are used, this is more difficult in the case of intentional cognitive tasks.

\section{Template Aging}

Most of the research papers included in this survey use data acquired in a single recording session, or in multiple sessions that are not separated significantly in time. To truly evaluate the potential of EEG based biometric systems, data acquisition should take place over time. Some papers using data across time to evaluate temporal effects are reviewed in Section $\mathrm{V}$ as part of the proposed novel framework for usability analysis.

\section{FEATURE EXTRACTION}

The choice of features is a crucial factor in determining the performance of an EEG-based biometric system. The search for suitable features for biometric recognition is governed by the nature of EEG signals: Features based on Fourier Transform (FT), are designed to capture the energy/spectra of the signals; other features are designed to capture the time-dependent information of the EEG signals; and wavelet-based features are developed to capture both the time and the frequency characteristics of EEG signals.

A review of recently published research focusing on using different feature extraction techniques for EEG biometrics is provided in this section. Each section begins with a brief description of the technique's rationale, followed by the related literature reports.

\section{A. Fourier Methods (PSD)}

One of the frequently employed features in EEG biometrics is the PSD. Conventionally, the PSD feature is computed from the FT of the time-domain signal, which indicates the spectral density distribution of the signal in the frequency domain. While the FT is well-defined when the signal is stationary [39], many bio-signals such as EEG signals are nonstationary. Therefore, usually the truncated FT $\hat{x}_{T}(\omega)$ over a finite interval $[0, T]$ is computed instead. Within that interval the signal is assumed to be stationary and the PSD, $S_{x}(\omega)$, of the signal $x(t)$ may be computed using (1), where $\hat{x}_{T}(\omega)$ : is the FT of $x(t)$ [39]

$$
S_{x}(\omega)=\lim _{T \rightarrow \infty} E\left[\left|\hat{x}_{T}(\omega)\right|^{2}\right] .
$$

As one of the most popular features in EEG biometrics, PSD was first employed in an identification scenario in 1999. Poulos et al. [6] used the spectral information of EEG signals the identities of four subjects, each having 45 three-minute recordings. Another group of 75 subjects was used as imposters in a simulated verification scenario. The voltage between $\mathrm{O} 2$ and $\mathrm{Oz}$ positions was used in their investigation. The power spectral values of three overlapping frequency bands were used as features: 7-10 Hz, 8-11 Hz, and 9-12 Hz. The features were fed into a learning vector quantization network for classification. A series of binary classifications were conducted: For each classification the data from one of the four subjects was compared with the 75 subjects-group in the dataset. The correct recognition rates (CRRs) ranged from $80 \%$ to $100 \%$.

PSD features have been used for identification within a larger population of 102 users in [35]. A series of power spectral features were developed using the multiple signal classification algorithm. Visual stimuli were employed to trigger informative EEG events for biometric recognition. A Manhattan distancebased $k$-NN classifier was used, reporting a maximum CRR of $98.12 \%$. However, the sensor system contained all the 61 electrodes in order to reach this performance level.

Safont et al. investigated the performance of EEG signals in an authentication scenario for a large database [40]. Data from two forehead electrodes (Fp1 and Fp2) were used; subjects were sitting in a dark room in a resting state during the data capture. A series of different features including maximum power of each channel were used for feature extraction. A population of 70 subjects was used for the verification evaluation, 20 of them acted as intruders and the rest were considered genuine. Genuine subjects had data recording durations ranging from 3 to $5 \mathrm{~min}$ 
and each intruder provided 2 minutes' data. A relatively good performance of $2.4 \%$ EER was reported.

Using low-cost sensors systems for EEG biometrics in conjunction with PSD features has also been explored [38]. The NeuroSky MindSet [13] single-sensor system has been used for data collection: different mental tasks were performed by each subject and repeated five times per session. Two sessions of data on separate days (40-50 min with multiple tasks per session) were captured; PSD coefficients of alpha band $(8-12 \mathrm{~Hz})$ and the beta band $(12-30 \mathrm{~Hz})$ were extracted; the cosine similarity was measured for classification in the verification scenario. The reported HTER was $1.1 \%$ by applying a "customized threshold" for 15 subjects.

The EEG Motor Movement/Imagery Dataset (containing data of four mental/cognitive tasks and two resting state tasks recorded using 64 electrodes) has been used for evaluating EEGbased biometric systems [25]. La Rocca et al. used only its baseline data (resting state of $1 \mathrm{~min}$, single session) for identification. A CRR of $100 \%$ was reported for a population of 109 subjects using the PSD-based feature ranged from 1-40 Hz.

\section{B. Autoregressive Model (AR)}

AR models have been another very popular feature since the early development of EEG biometrics [41]. An AR model is a time domain representation of a random process. The classic AR model for a random process $X_{t}$ is defined as follows [42]:

$$
X_{t}=c+\sum_{i=1}^{p} \varphi_{i} X_{t-i}+\varepsilon_{t}
$$

where $\varphi_{1}, \ldots, \varphi_{p}$ are the coefficients of the model, $c$ is a constant and $\varepsilon_{t}$ is the white noise. Equation (2) can be equivalently rewritten as (3) using the lag operator $B$

$$
X_{t}=c+\sum_{i=1}^{p} \varphi_{i} B^{i} X_{t}+\varepsilon_{t}
$$

as such, the signal $X_{t}$ can be represented by a series of AR coefficients $\varphi_{i}$ and white noise $\varepsilon_{t}$. The AR coefficients may reveal certain intrinsic characteristics of EEG signals, and therefore, suitable candidates as biometric features.

One of the early works using AR coefficients for EEG biometrics is reported by Paranjape et al. [43]. In their experiment, data from 40 subjects was obtained from one electrode (at the P4 position). The data recorded for each subject contained eight epochs (8.533 s per epoch) while subjects were in a resting state. A correct classification rate of $80 \%$ was achieved using a linear discriminate analysis (LDA) classifier. As an early EEG biometrics paper, the highlight of the experiment was the use of only one electrode for identification; however, the manual contamination removal that was used for data preparation maybe considered as a restriction on its applicability.

Using AR coefficients as features in a verification scenario was investigated by Riera et al. [44]. They reported a True Acceptance Rate of $96.6 \%$ in a database containing 87 subjects; 36 of which acted as impostors. Data capture was conducted while subjects were in a resting state. The recording length for each subject was between 2 to $4 \mathrm{~min}$; the genuine subjects were recorded at four sessions in different days whereas the impostors were recoded only once. Only two frontal electrodes (Fp1 and Fp2) were used and the frequencies above $50 \mathrm{~Hz}$ were removed. Fisher's discriminant analysis was used for classification and an EER of 3.4\% was reported. This result was obtained by using a number of different features (including PSD) and the outputs from separated classifiers were combined by fusing the scores provided by 28 Fisher's discriminant analysis classifiers with different features.

Campisi et al. investigated the recognition performance under an "Eyes Closed Resting Conditions" protocol [45]. The data was captured from 48 subjects. The sixth-order AR coefficients were used as features with a polynomial regression-based classifier. A genuine acceptance rate of $96.08 \%$ was reported by using a data capture device that comprised of 56 electrodes with a sampling frequency of $200 \mathrm{~Hz}$.

To further improve the performance of EEG biometric recognition, noise removal has also been taken into consideration in the preprocessing stage. Maiorana et al. [46] proposed a preprocessing method which combined independent component analysis and sample entropy to isolate and remove the artifacts. AR coefficients and PSD were employed as features for distance-based classification. For a population of 50 subjects the reported recognition rate using AR feature was about $95 \%$, using 19 electrodes.

AR-based features have also been tested using low-cost sensor system. Dan et al. [47] collected data from 13 healthy subjects using the NeuroSky MindSet single-sensor headset. Data from three sessions were captured on different days; subjects were in a resting state with eyes closed during the data collection. A recognition rate of $87 \%$ was achieved using an LDA classifier. Comparing the results of [38] which used the same sensor device, it seems the AR features may be better suited in an identification scenario, while the PSD-based features may be a better choice for verification.

\section{Wavelet Transform (WT)}

Another relatively new and increasingly popular feature extraction method used for EEG biometrics is the WT. Waveletbased features usually are derived from the wavelet coefficients $W T_{\psi}\{x\}(a, b)$, which can be computed using the following formula [48]:

$$
W T_{\psi}\{x\}(a, b)=<x, \psi_{a, b}>=\int_{R} x(t) \cdot \psi_{a, b}(t) d t
$$

where $x(t)$ is the time domain signal and $\psi_{a, b}(t)$ is the wavelet function. One advantage of wavelet-based methods is the flexibility of choosing the wavelet function $\psi_{a, b}(t)$ : Different wavelet functions with different scale, $a$, and shift, $b$, could be selected to suit specific application. Moreover, the WT can be designed to preserve signal content within specific ranges of both time and frequency in the wavelet domain which could potentially provide more relevant information in the feature space [48].

Gupta et al. [49] used wavelet packet decomposition (WPD) to extract three typical EEG bands (delta, theta, and gamma bands) with Daubechies (db4) and Coiflet (coif3) wavelets for 
biometric identification. The EEG signals were triggered by visual stimuli and the P300 ERP data from four subjects was captured using eight electrodes (around $200 \mathrm{~s}$ of recording per subject) and used for feature extraction. An identification performance of $85 \%$ was reported using a radial-based function Neural Network (NN) classifier.

Yang et al. [50], [51] did a series of investigations using WT for EEG biometrics. Wavelet-based algorithms were employed for both noise removal and feature extraction. A correct recognition accuracy of more than 90\% was achieved for 109 subjects using wavelet domain features, when combined with an entropy-based data-filtering algorithm [51].

\section{Hilbert-Huang Transform (HHT)}

The HTT is a relatively new algorithm initially reported in 1998 [52]. Though it was designed for dealing with nonstationary signals, its use for EEG biometrics is still relatively rare. The HHT is comprised of two main steps: 1) generating the intrinsic mode functions (IMF); in a process called empirical mode decomposition (EMD) and 2) performing the Hilbert Transform on each IMFs.

Given a signal $x(t)$, the effective algorithm of EMD can be summarized as follows [52], [53]:

1) Identify all extrema of $x(t)$.

2) Interpolate between minima and maxima, ending up with some "envelope" $e_{\min }(t)$ (and $e_{\max }(t)$ ).

3) Compute the average residual $m(t)=$ $\left(e_{\min }(t)+e_{\max }(t)\right) / 2$.

4) Extract the detail signal $d(t)=x(t)-m(t)$.

5) Iterate on the residual $m(t)$.

In practice, "the above procedure has to be refined by a 'sifting process' which amounts to first iterating steps 1)-4) upon the detail signal, until this latter approach a zero-mean according to some stopping criterion" [53]. Once this is achieved, the "detail" is considered as the effective IMF, the corresponding residual is computed and step 5) is applied [53].

The next step of the HHT algorithm is computing the Hilbert Transform. Denoting the resulting IMF(s) after EMD by $x(t)$, the HT of $x(t), y(t)$, can be computed using (5)

$$
y(t)=\frac{1}{\pi} p \int_{\tau}^{\infty} \frac{x(\tau)}{t-\tau} d \tau
$$

where

$$
P=\lim _{\varepsilon \rightarrow 0^{+}}\left[\int_{\tau}^{t-\varepsilon} \frac{x(\tau)}{t-\tau} d \tau+\int_{t-\varepsilon}^{\infty} \frac{x(\tau)}{t-\tau} d \tau\right] .
$$

$P$ is the Cauchy Principal Value defined by (6), due to the otherwise ill-defined function (5) while $\tau \rightarrow t$.

Kumari et al. [54] recently suggested using an EMD-based coefficient of variation (a parameter that relates to the standard deviation and mean of IMFs) as a feature for EEG biometric recognition. In a preliminary investigation, using data from only three subjects, they claimed that person identification performance is sensitive to the scalp regions from which the EEG signals are extracted.

Yang et al. [55] proposed the use of an improved EMD algorithm (Ensemble EMD [56]) for EEG feature extraction. Two novel features based on IMFs were developed. Using data from only one electrode $(\mathrm{Cz})$, recognition rates of $99 \%$ and $96 \%$ were reported for two publicly available databases both containing more than 100 subjects. These results, though could not yet establish the stability of EEG signals for person recognition over a long period of time, still proved the informative characteristics of EEG signals and the distinguishing power of the HHT in extracting those characteristics.

\section{E. Other Methods}

Besides the previously mentioned feature extraction approaches which are based on the three major signal transforms, other interesting features have been used for EEG biometrics. Singhal et al. [57] proposed a novel time domain peak matching algorithm using Visual Evoked Potentials (VEP) for identification. They reported a recognition rate of $78 \%$ for a ten subjects' database. From each subject 20 recordings (6 s each) were obtained. Half the data was used to train the system and the rest was used for testing.

Huang et al. [58] reported a simple but effective feature, tested using a relatively large VEP database (122 subjects). The proposed feature was the "equivalent root mean square (rms) values for each electrode signal over a $1 \mathrm{~s}$ period." This seems to capture the transient energy after each visual stimulus (1 s after the stimulus). As a time domain feature, its advantage was the simplicity of computation combined with a relatively high performance. The CRR reached $95.1 \%$ using the data from 64 electrodes for 116 subjects.

ERP was used for biometric recognition by Yearn et al. [59]. They claimed that showing the subject with self-face and nonself-face images would trigger different VEP signals, which may be used for person recognition. The "point-wise biserial correlation coefficients [60]" were employed as features; a CRR of $85.5 \%$ was reported using a self-collected database which contained 10 subjects. Only 18 electrodes were used for data analysis.

Phung et al. [61] used the Shannon Entropy (SE) of alpha, beta, and gamma bands' PSD as features. The performance was compared with conventional AR-based features and achieved similar CRRs (97.1\% versus $97.2 \%$, for SE and AR). The advantage of the SE feature was claimed to be a much faster identification speed (2.3-2.6 times faster than using the AR features).

Considering these reported research trends, most of the feature extraction methods were based on visual stimuli. The experimental results using several newer features, despite their novel designs, were either comparable with the conventional features such as AR coefficients and PSD ([58], [61]) or worse than them ([57], [59]).There is, therefore, a need for continued efforts to discover and develop new and more effective features for more robust EEG-based biometric recognition.

Wavelet-based and HHT-based features have shown significant potential in EEG biometric recognition, and it is, thus, interesting to develop further new features based on these transforms and evaluate them using larger databases (more than 100 subjects) extending over longer periods of time to also assess their template Aging performance. 


\section{FeATURE ClassificAtion}

After the choice of features, the next most important component for an EEG biometric system is the choice of the classifier and the strategy used to train it for a particular application. This section reviews some of the recent works using different feature classification techniques for EEG biometrics.

\section{A. $k$-Nearest Neighbor (k-NN) Algorithms}

One frequently used algorithm in EEG-based pattern classification is the $k-\mathrm{NN}$ algorithm. The basic principle of $k-\mathrm{NN}$ algorithms for classification is to compare the similarity or distance between the template feature samples and the query (or test) samples; the test set tends to find its $k$ nearest labeled samples in the training feature space and the decision can be made by a majority voting scheme: If the $k$ is set to 1 , the algorithm becomes a simple nearest neighbor classifier and the decision making is solely dictated by the label of that nearest neighbor's class [62].

The $k$-NN classifier directly uses the available training samples [63]. Therefore, the classification is sensitive to the local (geometric) distribution of the training samples in the feature space, such local sensitivity may potentially lead to the instability of performance [64]. Another shortcoming of the basic $k$-NN algorithm is that the density of the data clusters affect its performance and may lead to wrong decision making: depending on the parameter $k$, the decision may be biased toward the class with a high cluster density, and makes the $k$-NN classifier sensitive to noisy data [62]. To overcome this potential problem, some $k$-NN algorithms use the distance $(d)$ as a weight (conventionally $1 / d$ ) to improve the decision making [62].

Despite these shortcomings, $k$-NN does have one significant advantage: It does not have an explicit and distinctive training process. The available training samples (multidimensional feature vectors) only need to be stored together with their respective class labels and compared with the query samples directly. Such simplicity is a clear advantage over many other machine learning algorithms.

Yazdani et al. also employed the $k$-NN classifier for person identification using VEP data [65]. A 61-electrode sensor system captured the EEG data from 20 subjects. AR and PSD coefficients were used as features. They claimed a $100 \%$ accuracy rate using a 5-NN classifier: the parameter $k$ for $k$-NN was extensively tested from 1 to 100 . In these experiments the optimal number of $k$ for EEG biometric recognition tends to be relatively small.

Fei et al. used data captured with a single electrode (Fp1) from 40 subjects [66]. An identification accuracy of $97.5 \%$ was obtained using a $k$-NN classifier; Fisher's LDA was employed for feature reduction. The recognition performance achieved using a support vector machine (SVM) classifier was $81 \%$ in their experiments.

\section{B. Linear Discriminate Analysis}

According to its frequent appearance in the relevant literature and its high recognition performance, LDA is by far one of the most popular classifiers in EEG-based biometric classification. For a classification problem, LDA assumes that the conditional probability density functions of the classes are normally distributed with equal class covariance [67]. In the Bayesian framework, the optimal solution of a two-class problem can then be approached by computing the ratio of the interclass variance $\sigma_{\text {inter }}^{2}$ over the intra-class variance $\sigma_{\text {inter }}^{2}$ :

$$
S=\frac{\sigma_{\text {inter }}^{2}}{\sigma_{\text {intra }}^{2}} .
$$

The score $S$ of (7) is the likelihood ratio, which is used for optimizing the classification [68].

A closely related technique to LDA is the so-called Fisher's LDA, which also occasionally appears in EEG biometrics literatures. The only difference between Fisher's LDA and the conventional LDA is that the Fisher's LDA does not make some of the assumptions of LDA such as normally distributed classes or equal class covariance [68].

The work in [69] is one of the early reports which employed the LDA classifier to investigate the effectiveness of EEG signals for biometric person recognition. Publicly available data for five subjects performing mental/cognitive tasks were used. A series of different features were computed, including AR coefficients, channel spectral powers, interhemispheric channel spectral power differences, and interhemispheric channel linear complexity. A standard PCA algorithm was employed for feature dimension reduction and the resulting features were fed to a LDA classifier. The reported results indicated a minimum average recognition error of $0 \%$.

In [70], an LDA classifier was employed, and the spectral power, the maximum power, and the frequency of maximum power in the alpha band were used as features. Two sessions of data from four subjects were collected using "one bipolar channel (O1A2)," and the time interval between the sessions ranged from 10 days to 5 months. The "authentication performance" was reported as $98.33 \%$ with test recordings of $20 \mathrm{~s}$ duration. The results suggest robustness to template Aging (as opposed to the report in [71], which showed a $10.9 \%$ degradation of the identification rate in the repeatability investigation).

\section{Artificial Neural Networks (ANNs)}

ANN forms a family of statistical learning algorithms inspired by biological neural networks (in particular the brain). These can be used to estimate or approximate functions that may depend on a large number of generally unknown inputs [62]. In general, an ANN can be viewed as a network of simple classifiers; each classifier in the network is an activation function which only responds to the input from its previous neuron (function). The results of these functions are weighted and fused before they reach the final decision making phase.

ANNs have been popular classifiers for EEG biometrics since this modality was first explored [6]. The work reported in [72] is one of the early studies which employed this kind of classifier for EEG-based person identification. In that study a "multilayer perceptron NN with a single hidden layer" was used for classifying the data obtained from 20 subjects. A headset with 61 
electrodes was used to capture $40 \mathrm{~s}$ of VEP data (in a single recording session). An identification accuracy of $99.06 \%$ was reported by computing the conventional PSD-based features from gamma band data $(32-40 \mathrm{~Hz})$.

Using a single electrode $(\mathrm{Cz})$ in an ANN-based identification scenario was investigated in [73]. Data from only three subjects was obtained and filtered to retain the frequency ranges from 1 to $12 \mathrm{~Hz}$. The reconstructed time domain data was fed directly to a single hidden layer ANN, trained by the data from five trials (1 s of visual stimulus per trial), and an accuracy of $100 \%$ was reported.

Using a low-cost sensor system for VEP data collection was investigated by Gui et al. [74]. They reported using a six midline electrode system ("EASY CAP" [75]) in an identification scenario for 32 subjects. Only the data obtained by the electrode placed in the $\mathrm{Oz}$ location was employed for feature extraction. The WPD was applied for noise-removal; the mean, SD and entropy of the resulting wavelet coefficient were computed as features. The VEP data was collected during the subjects were reading an unconnected list of texts. Each recording lasted for $1.1 \mathrm{~s}$, the overall recording length contained about $50 \mathrm{~s}$ per subject. $70 \%$ of the obtained data was used to train a "feed-forward, back-propagation, multilayer perceptron" neural network and the rest of the data was used for testing the recognition accuracy. A performance of around $90 \%$ in a one against the rest identification scenario (binary classification) was reported. They also reported that, in a conventional one-to-many identification scenario (32 classes) the obtained accuracy was less than $11 \%$, which may indicate the low-cost single sensor system was still not reliable enough for realistic biometric scenarios.

The quantity of data and time required for training ANNs is a major concern for effective deployment. The reported researches on EEG biometrics usually employ ANNs with simple structures (e.g., a single hidden layer). With the improvement of computational power, i.e., faster central processing units, and the employment of graphics processing units, the use of more complex networks, such as recurrent and convolutional neural networks have shown great potential in pattern recognition [76]. The concept of "deep learning" has been quite successfully implemented in many pattern recognition fields, including handwriting recognition and fast image processing [77]. Such classifiers hold great promise for application in EEG-based biometrics recognition.

\section{Kernel Methods}

Kernel-based classifiers were proposed as early as the 1960s, with the invention of the kernel perceptron [77], [78]. These algorithms became prominent with the popularity of the SVM in the $1990 \mathrm{~s}$, when the SVM was found to be competitive with neural networks on tasks such as handwriting recognition [79].

The SVM classifier integrates the notion of supporting vectors and kernel tricks. Given a set of training samples, SVM is designed to find the "maximum-margin hyper plane" in feature space which indicates the maximum gap between two classes: The feature vector(s) which form the hyper plane(s) are called the "supporting vectors" [79]. However, it often happens that the available data are not linearly separable in the original feature space. Therefore, it was proposed by Vapnik et al. [79] that the original finite-dimensional space may be mapped into a space with much higher-dimensionality to make the separation easier in that space. The mapping of the original space onto higher/infinite spaces is called the "kernel trick" [78]. Some commonly used functions (kernels) for this mapping in SVM are polynomial functions, the Gaussian radial basis function, and the hyperbolic tangent function [79].

In the field of EEG biometrics, the use of SVM classifiers has also received attention. Using conventional AR coefficients features for classification, results were presented using three classifiers, namely LDA, back-propagation Neural Network, and SVM in [80]. The reported accuracies, using a small publicly available database with three subjects and using five electrodes, indicate that ANN and SVM provided comparable performance (identification accuracy ranges from $80.8 \%$ to $84.0 \%$ ) and LDA outperformed both of them by more than $5 \%(89.5 \%)$.

In [81], the SVM classifier was used with the data obtained using a low-cost sensor system (Emotiv [16] with 14 electrodes) for five subjects. Each of them "performed four different mental tasks" with an overall recording length of 150 s. A series of different features were extracted: sixth order autoregressive (AR) coefficients, PSD, and total power in five frequency bands, interhemispheric power differences and interhemispheric linear complexity. These features were fed into a one-versus-all linear SVM [82]: For every subject, the data from four tasks (10 s per tasks) were combined and used for testing the performance, the rest of the data was used for training the SVM. Classification accuracy of $100 \%$ was reported through the combination of two voting rules by computing the 1 minus the averaged HTER.

To summarize the characteristics of the classifiers reviewed in this section, those based on the $k-\mathrm{NN}$ algorithm tend to require the least computation time for model training, while maintaining acceptable recognition performance [83]. However, the testing for $k$-NN can be time-consuming as it compares the features exhaustively during the process. Compared to $k$-NN classifiers, the SVM and ANN classifiers are more complex and they have the potential to achieve much better performance in EEG biometric recognition. Especially with the recent development of "deep learning" algorithms, it is worth investigating the application of improved ANN techniques to EEG biometric recognition. The challenge here is the need for substantial training data that is required to train such large networks. The LDA algorithm has demonstrated very good performance amongst the results reviewed, and unlike the SVM and ANN classifiers, which can be quite time-consuming in training, has a satisfactory tradeoff between training/testing time and classification performance.

\section{USABILITY INVESTIGATION OF THE REPORTED RESULTS}

The use of EEG data for person recognition has been explored since 1998 and more than 100 papers in this field are published. Most of these papers were focused on reporting the performance using conventional accuracy metrics (in both the identification and verification scenarios). However, to objectively assess an EEG biometric recognition system and establish its potential 
suitability for real-life applications, the performance of EEGbased biometrics system needs to be evaluated based on more factors than the recognition accuracy only. In this section, five factors affecting usability in practice are investigated and combined to provide one single metric to provide an indication of the potential usability of the EEG-based biometric systems.

Besides the conventional measures of recognition accuracy, there are at least another four factors which should be taken into consideration for assessing the practical usability of any reported EEG-based recognition systems:

1) number of the subjects for which the system was designed or tested on $(N)$;

2) number of the electrodes employed $(K)$;

3 ) the recording duration of the training set $(T r)$; and

4) recording duration of the test set (Te).

The number of electrode(s) used for data collection has a considerable impact on the usability of the EEG-based biometric system: A large number of electrodes may increase the difficulty in deploying the system in real-life scenarios. The length of the EEG recordings employed for system training, and particularly the processing time required for person recognition are quite influential factors as well. Number of the subjects employed indicates the effectiveness of the biometric system in large scale deployments. Training and test durations (in second) are proposed to be used here instead of the number of samples, as in the actual application it is the time spent by user(s) and the computational efficiency that matters.

Including the percentage $C R R$, five factors in total may be considered together in evaluating a system. Of these five factors, $K, T r$, and $T e$ should ideally be as small as possible for practical deployment; $N$ and $C R R$ should be as large as possible. Therefore, the following metric (8) is proposed to combine these factors and produce an overall score, $U$, to evaluate the system:

$$
U=\frac{N \times \mathrm{CRR}}{T r+K \times T e}
$$

This score, which indicates the overall "effectiveness" or usability of an EEG biometric system, can therefore be used to provide a meaningful comparison of the systems that have been covered in this review: The higher the value of $U$, the better the system's overall usability. The time required for the user to provide data in normal use is represented by the testing time, $T e$, and is considered to have a greater weight in establishing the usability of the system compared with the one-off time required for training, $\operatorname{Tr}$ : Since the tests may be conducted multiple times, the number of electrodes $K$ is used as the weighting parameter for $T e$ in (8). This is because the greater the number of electrodes, the higher is the effort and cost needed to gather the data, hence lower usability of the system.

In the following sections, the proposed metric, $U$, is used to compare the systems covered in the review. These are grouped by the different feature types that have been used for classification. As it was shown in Section III that the choice of feature is crucial for EEG-based biometric systems, it is therefore, interesting to investigate the usability scores for the different feature
TABLE II

IDENTIFICATION COMPARISONS OF THE REPORTS USING PSD FOR FEATURE EXTRACTION

\begin{tabular}{lcccccc}
\hline \hline Reports & $N$ & $K$ & $\operatorname{Tr}(s)$ & $\operatorname{Te}(s)$ & $C R R(\%)$ & $U$ \\
\hline Poulos et al. [41] & 4 & 1 & 3600 & 4500 & 95 & 0.05 \\
Palaniappan et al. [29] & 10 & 61 & 20 & 20 & 95 & 0.77 \\
Palaniappan [31] & 20 & 61 & 20 & 20 & 94.18 & 1.52 \\
Palaniappan [72] & 20 & 61 & 20 & 20 & 99.06 & 1.59 \\
Ravi et al. [32] & 40 & 61 & 20 & 20 & 96.63 & 3.12 \\
Palaniappan et al. [34] & 40 & 35 & 20 & 20 & 98.56 & 5.48 \\
Palaniappan et al. [35] & 102 & 61 & 20 & 20 & 98.12 & 8.07 \\
Sun Shiliang [84] & 9 & 15 & 540 & 270 & 95 & 0.19 \\
F.Su et al. [85] & 40 & 1 & 1800 & 1800 & 97.5 & 1.08 \\
Hema et al. [86] & 15 & 2 & 160 & 40 & 89.95 & 5.62 \\
Zhao et al. [87] & 10 & 1 & 100 & 50 & 96.77 & 6.45 \\
Quintela et al. [88] & 70 & 8 & 45 & 45 & 99.1 & 17.13 \\
La Rocca et al. [25] & 108 & 56 & 50 & 10 & 100 & 17.70 \\
Bai et al. [89] & 20 & 32 & 10.8 & 1.2 & 97.25 & $\mathbf{3 9 . 5 3}$ \\
\hline \hline
\end{tabular}

TABLE III

IDENTIFICATION COMPARISONS OF THE REPORTS USING AR COEFFICIENTS FOR FEATURE EXTRACTION

\begin{tabular}{lcccccc}
\hline \hline Reports & $N$ & $K$ & $\operatorname{Tr}(s)$ & $\operatorname{Te}(s)$ & $C R R(\%)$ & $U$ \\
\hline Poulos et al. [90] & 4 & 1 & 3600 & 4500 & $72 \sim 84$ & 0.04 \\
Poulos et al. [41] & 4 & 1 & 1800 & 6300 & $91 \sim 97$ & 0.05 \\
Paranjape et al. [43] & 40 & 1 & 34 & 34 & 80 & 47.06 \\
Poulos et al. [91] & 4 & 1 & 3600 & 4500 & 99.5 & 0.05 \\
Palaniappan et al. [36] & 4 & 6 & 50 & 50 & 99.04 & 1.13 \\
Palaniappan et al. [69] & 5 & 6 & 50 & 50 & 99.88 & 1.43 \\
Yazdani et al. [65] & 20 & 61 & 89 & 1 & 100 & 13.33 \\
F.Su et al. [85] & 40 & 1 & 1800 & 1800 & 97.5 & 1.08 \\
Zhao et al. [87] & 10 & 1 & 100 & 50 & 96.77 & 6.45 \\
Campisi et al. [45] & 48 & 3 & 43 & 17 & 96.08 & $\mathbf{4 9 . 0 6}$ \\
Kostilek [71] & 9 & 53 & 45 & 15 & 98 & 1.05 \\
La Rocca et al. [92] & 45 & 3 & 43 & 17 & 98.73 & 47.26 \\
Dan et al. [47] & 13 & 1 & 9360 & 4680 & 87 & 0.08 \\
\hline \hline
\end{tabular}

types separately to explore the effectiveness before the feature classification.

\section{A. PSD-Based Systems}

As it was stated in Section III.A, PSD features have been amongst the most used for EEG biometric recognition. Table II compares, with the help of the proposed metric, the effective usability of some reported systems that employ these features. The order of the listed papers follows the year of the publication in ascending order. As it can be observed in Table II, the scores generally tend to increase as the year of publication increase, indicating a trend of improving usability of EEG biometric systems. The highest score listed in the table reached $S=39.53$, which indicates the best overall performance using PSD-based features amongst the papers covered in this review.

\section{B. AR-Based Systems}

Feature extraction based on AR coefficients is another popular approach in EEG biometrics (Section III.B); some relevant reports with experimental details are compared in Table III using the proposed usability metric. Based on this metric, the best 
TABLE IV

IDENTIFICATION COMPARISONS OF THE REPORTS USING OTHER ALGORITHMS FOR FEATURE EXTRACTION

\begin{tabular}{lcccccc}
\hline \hline Reports & $N$ & $K$ & $\operatorname{Tr}(s)$ & $T e(s)$ & $C R R(\%)$ & $U$ \\
\hline Gupta et al. [49] & 4 & 8 & 132 & 68 & 85 & 0.51 \\
Yang et al. [93] & 18 & 8 & 240 & 60 & 97.4 & 2.44 \\
Yeom et al. [59] & 10 & 18 & 360 & 40 & 85.5 & 1.14 \\
Yang et al. [51] & 109 & 8 & 240 & 60 & 90 & 13.63 \\
La Rocca et al. [94] & 36 & 3 & 40 & 20 & 99.69 & 35.89 \\
Yang et al. [50] & 50 & 5 & 114 & 6 & 95.5 & 33.16 \\
La Rocca et al. [25] & 108 & 9 & 50 & 10 & 100 & 77.14 \\
Phung et al. [61] & 40 & 23 & 800 & 400 & 97.1 & 0.39 \\
Yang et al. [55] & 105 & 1 & 96 & 24 & 99 & $\mathbf{8 6 . 6 3}$ \\
\hline \hline
\end{tabular}

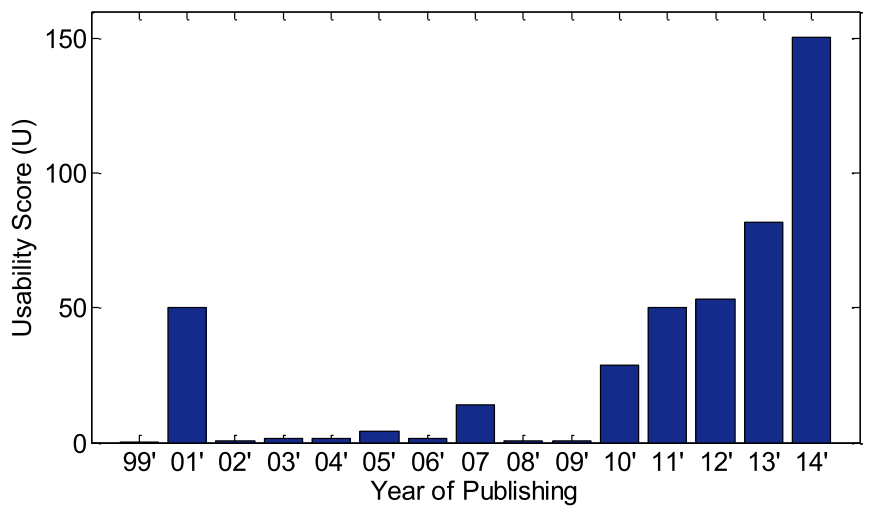

Fig. 2. Yearly plot of the usability scores.

performed system using the AR features performed better than the best system using the PSD-based features amongst the papers reviewed in this survey.

\section{Other Feature-Based Systems}

In this section, papers reporting other features for use in EEG biometrics are compared using the proposed metric in Table IV. The usability score $U=89.93$ was reported using a waveletbased feature, which is the best-reported performance amongst the EEG biometrics papers covered in this survey.

The yearly cumulative usability scores of the reported EEG biometric system are shown by Fig. 2. The usability scores of the reported systems are summed per year. It is noticed the score has a sharp increase in the year 2001. A possible reason of this may have to do with the use of a manual data cleaning process in that experiment: "the muscle (EMG), cardiac (ECG), or other noise signals were removed" by "a trained neurologist" [43]. The usability of EEG biometric appears to be consistently increased in the past 5 years, a fact that is reflected by both the growing $r$ research effort in this area and the closer attention to usability issues in system design.

In summary, the usability scores of the reported systems seem to indicate that some of the early reported EEG biometric systems may have been focusing only on the recognition performance and ignored other important factors, such as the number of electrodes and the recording length. The proposed usability metric reveals the gap between the laboratory context where these systems are developed and the real-world environments of target applications for EEG biometric recognition (considering the reported high accuracies and some systems with low usability scores). However, due to the limited number of the available reports related to template Aging, it has not so far been possible to systematically consider the template Aging effect in the relative evaluation of different EEG biometric recognition systems.

Indeed, in biometric person recognition the template Aging is an important consideration that may limit the usability of biometric systems in terms of the need for frequent acquisition of new enrolment data. The comparisons reported in these tables were either obtained using single session EEG data or trained using concatenated data from multiple sessions. Therefore, the factor of template Aging has been excluded from the proposed metric. The following section is devoted to the issue of template Aging and suggests a way forward to extend the usability evaluation framework to the analysis of template Aging effects where test data is available.

\section{Template Aging}

Template Aging is an important phenomenon that impacts the effectiveness and usability of biometric systems as the time between enrolment and subsequent use of a biometric systems is increased. Having a better understating of template Aging is of particular importance for EEG biometrics as this is a relatively new modality and its performance characteristics with the passage of time is yet to be firmly established.

In this section, we explore how the usability metric defined in (8) may be extended to the case where data is available for more than one set of tests separated in time. In this way, it is hoped that the impact of template Aging may be incorporated in the relative ranking of different EEG-based biometric systems as more data becomes available. However, there are still only a very few studies published where performance evaluations are reported at different time intervals. We, therefore, start by mentioning two of these studies that could be the basis for presenting the extension to our usability analysis.

Template Aging effects when using mental-tasks with long time interval between training and testing has been reported in [71], where experiments were conducted using 53 electrodes and 9 subjects (using actual and imagined motor tasks) from two sessions, with a time interval of approximately 1 year. Using part of the data from the first session for training and the rest of the first session's data as the test set, the CRR reached 98\%; whereas using the first session's data for training and data of the second session for testing, the CRR was reduced to $87.1 \%$.

ERP data captured from a single sensor at the $\mathrm{Oz}$ position was used for template Aging analysis for biometric recognition by Blondet et al. [95]. They conducted the experiments with 15 subjects for multiple sessions with a time interval between the first two sessions ranging from 5 to 40 days. Out of those participants, nine returned for a third time, after 134-188 days from the first session (mean: 156 days or 5 months). The time domain cross-correlation was computed for feature extraction. An "average accuracy of 0.99 was reported for the first session, 0.93 for the second session, and 0.84 for the third session." 
TABLE V

MULTISESSION USABILITY ESTIMATION

\begin{tabular}{lcc}
\hline \hline Comparison $U_{0}$ & $U_{T}$ & $T$ (month) \\
\hline System 1 [95] 1.375 & 1.29 & 0.5 \\
System 2 [71] 0.27 & 0.24 & 12 \\
\hline \hline
\end{tabular}

Recently, Maiorana et al. [96] reported an EEG biometric recognition system with promising results which addressed the issue of single-session and multisession. Their experiments using the data acquired in a time span of approximately one month and a half, both PSD and AR based features were investigated, 50 healthy subjects in resting state conditions were involved the experiments.

Given that these reports provided at least two performance measurements at a significant time separation, we propose the following extension to our usability analysis in order to be able to compare these systems, while taking into account the effect of template Aging.

A new estimator $U_{t}$ is proposed that will predict the usability score for a system at any given time $t$ given the usability scores computed using (8) at two different points separated in time separated by $T$. In the absence of substantial data to warrant a more complex model, here we make a working assumption that a linear model would be adequate for accounting for the change in performance, and therefore usability, as a function of time

$$
U_{t}=U_{0}-t \frac{U_{0}-U_{T}}{U_{T}}
$$

The new estimator is defined by (9), where $U_{0}$ and $U_{T}$ represent the usability score for the first session and the second session, respectively. $U_{t}$ indicates the usability score at any particular time $t$ for a given recognition system. Therefore, it is possible to plot and compare the change in usability score for two or more EEG biometric systems as long as at least two recognition rates in different sessions are reported. For cases where data for more than two sessions are available, it may be possible to use a best fit line (or polynomial) to model the change in performance as a function of time.

To illustrate the proposed usability analysis framework, we use the data from two of the papers mentioned above ([81] and [107]), where multisession recognition rates are reported together with clear indications of time separation. The usability scores at different time separations for these two systems are computed using (8) and recorded in Table V.

In order to compare these two systems' usability score at a given time, these two systems ([95] and [71]) are represented by "System 1" and "System 2," respectively in Fig. 3 where their $U_{t}$ is plotted as a function of $t$.

Fig. 3 shows the trend in the degradation of the two systems' usability scores during 12 months, assuming a linear model. System 1 is seen to provide much better usability in the first few months. However, after approximately 7 months the usability of System 2 is seen to surpass that of System 1.

Clearly, this analysis framework can be significantly enhanced as more data at different time intervals becomes available

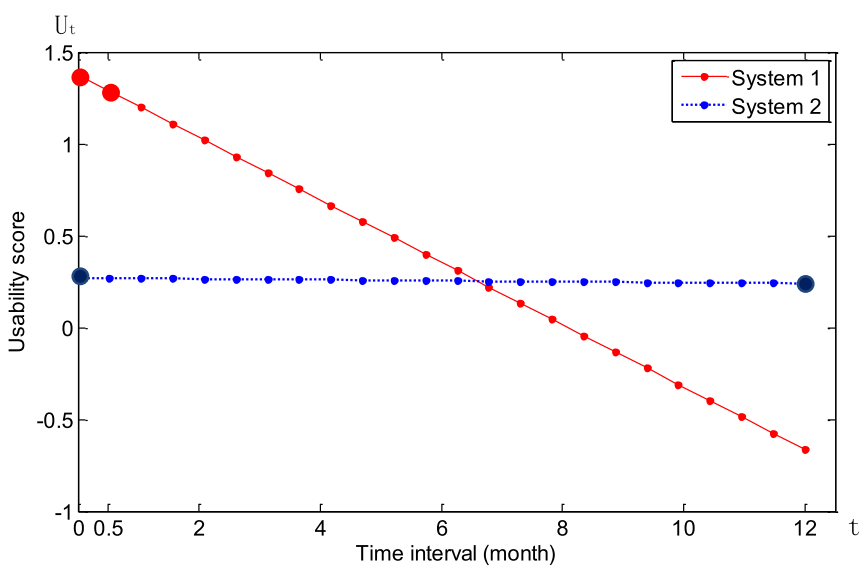

Fig. 3. Illustration of the multisession usability analysis.

making it possible to produce better models of the template Aging effects. However, given the current quantity and quality of the data available, it presents a first approximation at trying to establish the relative merit of published systems.

The results reported so far suggest that while template Aging may result in a decrease in accuracy and usability in EEGbased biometric systems, the impact of Aging may be limited. However, these are for based on tests using few subjects and relatively short temporal separations. More research is required to quantify the nature and extent of template Aging effects in EEG biometrics.

\section{CONCLUSION AND FutURE WORK}

This paper provided a review of the recent literature on EEGbased biometric person recognition. As this is a relatively new biometric modality, the literature has been focused on establishing the presence of biometric information in EEG signals. The use of EEG biometrics in real-world application scenario would need much more research to address the shortcomings of the work done to date especially with regards to the quantity and nature of data available for system evaluations.

The search for better acquisition systems, signal preprocessing, feature extraction, and classification should target the goal of reducing the sensor costs (electrode numbers) and quantity of data needed for training and testing while maintaining high recognition rates. Quantifying and ameliorating the effects of template Aging in EEG biometrics remains an important area for further research.

\section{REFERENCES}

[1] E. Niedermeyer and F. H. L. da Silva, Electroencephalography: Basic Principles, Clinical Applications, and Related Fields. Philadelphia, PA, USA: Lippincott Williams \& Wilkins, 2005.

[2] B. E. Swartz, "The advantages of digital over analog recording techniques," Electroencephalogr. Clin. Neurophysiol., vol. 106, no. 2, pp. 113-117, Feb. 1998.

[3] A. Coenen, E. Fine, and O. Zayachkivska, "Adolf Beck: A forgotten pioneer in electroencephalography," J. Hist. Neurosci., vol. 23, no. 3, pp. 276-86, Jan. 2014.

[4] L. F. Haas, "Hans Berger (1873-1941), Richard Caton (1842-1926), and electroencephalography," J. Neurol. Neurosurgery Psychiatry, vol. 74, no. 1, pp. 9-9, Jan. 2003. 
[5] S. Aydin, H. M. Saraoğlu, and S. Kara, "Log energy entropy-based EEG classification with multilayer neural networks in seizure," Ann. Biomed. Eng., vol. 37, no. 12, pp. 2626-30, Dec. 2009.

[6] M. Poulos, M. Rangoussi, and N. Alexandris, "Neural network based person identification using EEG features," in Proc. 1999 IEEE Int. Conf. Acoust., Speech, Signal Process., 1999, vol. 2, pp. 1117-1120.

[7] P. Campisi and D. La Rocca, "Brain waves for automatic biometric based user recognition," IEEE Trans. Inf. Forensics Security, vol. 9, no. 5, pp. 782-800, May 2014.

[8] S. N. Abbas, M. Abo-Zahhad, and S. M. Ahmed, "State-of-the-art methods and future perspectives for personal recognition based on electroencephalogram signals," IET Biometrics, vol. 4, pp. 179-190, Mar. 2015.

[9] R. W. Homan, J. Herman, and P. Purdy, "Cerebral location of international 10-20 system electrode placement," Electroencephalogr. Clin. Neurophysiol., vol. 66, no. 4, pp. 376-382, Apr. 1987.

[10] "EEG Motor Movement/Imagery Dataset." [Online]. Available: http://www.physionet.org/pn4/eegmmidb/. Accessed on: Apr. 20, 2015.

[11] G. Chatrian, "Ten percent electrode system for topographic studies of spontaneous and evoked EEG activity," Amer. J. Electroencephalogr. Technol., vol. 25, pp. 83-92, 1985.

[12] W. O. Tatum, "Ellen R. Grass lecture: Extraordinary EEG," Neurodiagn. J., vol. 54, pp. 3-21, Jan. 2014.

[13] "Neurosky Products." [Online]. Available: http://store.neurosky. com/products. Accessed on Apr. 20, 2015.

[14] G. Schalk, D. J. McFarland, T. Hinterberger, N. Birbaumer, and J. R. Wolpaw, "BCI2000: A general-purpose brain-computer interface (BCI) system," IEEE Trans. Biomed. Eng., vol. 51, no. 6, pp. 1034-43, Jun. 2004.

[15] schalklab, "BCI 2000." [Online]. Available: http://www.schalklab. org/research/bci2000. Accessed on May 21, 2015.

[16] "Emotiv." [Online]. Available: http://emotiv.com/. Accessed on Apr 20, 2015.

[17] "MindWave." [Online]. Available: http://store.neurosky.com/products/ mindwave-1. Accessed on Jul 10, 2015.

[18] "OCZ NIA Neural Impulse Actuator: Amazon.co.uk: Computers \& Accessories." [Online]. Available: http://www.amazon.co.uk/OCZ-NIA-NeuralImpulse-Actuator/dp/B00168VU4U. Accessed on Jul 10, 2015.

[19] "Melon Headband." [Online]. Available: http://www.thinkmelon.com/. Accessed on Jul 10, 2015.

[20] "HiBrain." [Online]. Available: http://www.hyperneuro.cn/. Accessed on Jul 10, 2015.

[21] "FocusBand - Mind Training Headset." [Online]. Available: http://www. ifocusband.com/. Accessed on Jul 10, 2015.

[22] "MUSE | Meditation Made Easy." [Online]. Available: http://www. choosemuse.com/. Accessed on Jul 10, 2015.

[23] "OpenBCI." [Online]. Available: http://www.openbci.com/. Accessed on Jul 10, 2015.

[24] “Aurora Dream Headband." [Online]. Available: https://iwinks.org/. Accessed on Jul 10, 2015.

[25] D. LA Rocca et al., "Human brain distinctiveness based on EEG spectral coherence connectivity," IEEE Trans. Biomed. Eng., vol. 61, no. 9, pp. 2406-2412, Sep. 2014.

[26] M. Fraschini, A. Hillebrand, M. Demuru, L. Didaci, and G. L. Marcialis, "An EEG-based biometric system using eigenvector centrality in resting state brain networks," IEEE Signal Process. Lett., vol. 22, no. 6, pp. 666-670, Jun. 2015.

[27] S. J. Luck, An Introduction to the Event-Related Potential Technique. Cambridge, MA, USA: MIT Press, 2014.

[28] E. Donchin, K. M. Spencer, and R. Wijesinghe, "The mental prosthesis: assessing the speed of a P300-based brain-computer interface," IEEE Trans. Rehabil. Eng., vol. 8, no. 2, pp. 174-179, Jun. 2000.

[29] R. Palaniappan and P. Raveendran, "Individual identification technique using visual evoked potential signals," Electron. Lett., vol. 38, no. 25, Dec. 2002, Art. no. 1634.

[30] J. G. Snodgrass and M. Vanderwart, "A standardized set of 260 pictures: Norms for name agreement, image agreement, familiarity, and visual complexity," J. Exp. Psychol. Hum. Learn. Mem., vol. 6, no. 2, pp. 174-215, 1980.

[31] R. Palaniappan, "A new method to identify individuals using signals from the brain," in Proc. Joint Conf. 4th Int. Conf. Inf., Commun. Signal Process., 4th Pacific Rim Conf. Multimedia, 2003, pp. 1442-1445.

[32] R. Palaniappan, "Recognising individuals using their brain patterns," in Proc. 3rd Int. Conf. Inf. Technol. Appl., 2005, vol. 2, pp. 520-523.
[33] R. Palaniappan, "Vision related brain activity for biometric authentication," in Proc. 32nd Annu. Conf. IEEE Ind. Electron., 2006, pp. 3227-3231.

[34] R. Palaniappan and D. P. Mandic, "EEG based biometric framework for automatic identity verification," J. VLSI Signal Process. Syst. Signal Image. Video Technol., vol. 49, no. 2, pp. 243-250, Jun. 2007.

[35] R. Palaniappan and D. P. Mandic, "Biometrics from brain electrical activity: a machine learning approach," IEEE Trans. Pattern Anal. Mach. Intell., vol. 29, no. 4, pp. 738-42, Apr. 2007.

[36] R. Palaniappan, "Identifying individuality using mental task based brain computer interface," in Proc. 3rd Int. Conf. Intell. Sensing Inf. Process., 2005, pp. 238-242.

[37] S. Marcel and J. D. R. Millán, "Person authentication using brainwaves (EEG) and maximum a posteriori model adaptation.," IEEE Trans. Pattern Anal. Mach. Intell., vol. 29, no. 4, pp. 743-52, Apr. 2007.

[38] J. Chuang, H. Nguyen, C. Wang, and B. Johnson, "I think, therefore I am: Usability and security of authentication using brainwaves," in Proc. Workshop Usable Security, 2013, vol. 424422, pp. 1-16.

[39] A. Oppenheim, Discrete-Time Signal Processing. Englewood Cliffs, NJ, USA: Prentice-Hall, 1989.

[40] G. Safont, A. Salazar, A. Soriano, and L. Vergara, "Combination of multiple detectors for EEG based biometric identification/authentication," in Proc. 2012 IEEE Int. Carnahan Conf. Security Technol., 2012, pp. 230-236.

[41] M. Poulos, M. Rangoussi, V. Chrissikopoulos, and A. Evangelou, "Parametric person identification from the EEG using computational geometry," in Proc. 6th IEEE Int. Conf. Electron., Circuits Syst., 1999, vol. 2, no. 2, pp. 1005-1008.

[42] H. Akaike, "A new look at the statistical model identification," IEEE Trans. Automat. Control, vol. 19, no. 6, pp. 716-723, Dec. 1974.

[43] R. B. Paranjape, J. Mahovsky, L. Benedicenti, and Z. Koles', “The electroencephalogram as a biometric," in Proc. Canadian Conf. Elect. Comput. Eng., 2001, vol. 2, pp. 1363-1366.

[44] A. Riera, A. Soria-Frisch, M. Caparrini, C. Grau, and G. Ruffini, "Unobtrusive biometric system based on electroencephalogram analysis," EURASIP J. Adv. Signal Process., vol. 2008, no. 1, 2008, Art. no. 143728.

[45] P. Campisi et al., "Brain waves based user recognition using the "Eyes Closed Resting Conditions ' protocol,' in Proc. 2011 IEEE Int. Workshop Inf. Forensics Security, 2011, pp. 16-19.

[46] E. Maiorana, J. Solé-Casals, and P. Campisi, "EEG signal preprocessing for biometric recognition," Mach. Vis. Appl., vol. 27, no. 8, pp. 1351-1360, 2016.

[47] Z. Dan, Z. Xifeng, and G. Qiangang, "An Identification System Based on Portable EEG Acquisition Equipment," in Proc. 3rd Int. Conf. Intell. Syst. Design Eng. Appl., 2013, pp. 281-284.

[48] I. Daubechies, Ten Lectures on Wavelets, vol. 61. Philadelphia, PA, USA: Society Ind. Appl. Math., 1992.

[49] C. N. Gupta, Y. U. Khan, R. Palaniappan, and F. Sepulveda, "Wavelet framework for improved target detection in oddball paradigms using P300 and gamma band analysis," Biosensors, Data Acquisition, Process. Control, vol. 14, no. 2, pp. 61-67, 2009.

[50] S. Yang and F. Deravi, "Wavelet-based EEG preprocessing for biometric applications," in Proc. 4th Int. Conf. Emerg. Security Technol., Sep. 2013, pp. 43-46.

[51] S. Yang and F. Deravi, "Quality filtering of EEG signals for enhanced biometric recognition," in Proc. Int. Conf. Biometrics Special Interest Group, 2013, pp. 1-4.

[52] N. E. Huang et al., "The empirical mode decomposition and the Hilbert spectrum for nonlinear and non-stationary time series analysis," Proc. Roy. Soc. A, Math. Phys. Eng. Sci., vol. 454, no. 1971, pp. 903-995, Mar. 1998.

[53] P. Flandrin, G. Rilling, and P. Gonçalvés, "Empirical mode decomposition as a filter bank," Signal Process. Lett., vol. 11, no. 2, pp. 112-114, 2004.

[54] P. Kumarii, S. Kumar, and A. Vaishi, "Feature extraction using emprical mode decomposition for biometric system," in Proc. Signal Propag. Comput. Technol., 2014, pp. 283-287.

[55] S. Yang and F. Deravi, "Novel HHT-based features for biometric identification using EEG signals," in Proc. 22nd Int. Conf. Pattern Recognit., Aug. 2014, pp. 1922-1927.

[56] N. E. Huang, Z. Wu, S. R. Long, K. C. Arnold, X. Chen, and K. Blank, "On instantaneous frequency," Adv. Adapt. Data Anal., vol. 1, no. 2, pp. 177-229, 2009.

[57] G. K. Singhal and P. RamKumar, "Person identification using evoked potentials and peak matching," in Proc. Biometrics Symp., 2007, pp. 1-6. 
[58] X. Huang, "Human identification with electroencephalogram ( EEG ) signal processing," in Proc. Int. Symp. Commun. Inf. Technol., 2012, pp. 1021-1026.

[59] S.-W. L. Seul-Ki Yearn and Heung-II Suk, "EEG-based Person authentication using face stimuli," in Proc. Int. Winter Workshop Brain-Comput. Interface, 2013, pp. 58-61.

[60] B. Blankertz, S. Lemm, M. Treder, S. Haufe, and K. Müller, "Single-trial analysis and classification of ERP components-A tutorial," Neuroimage, vol. 56, no. 2, pp. 814-825, 2011.

[61] D. Phung, D. Tran, W. Ma, P. Nguyen, and T. Pham, "Using Shannon entropy as EEG signal feature for fast person identification," in Proc. Eur. Symp. Artif. Neural Netw., 2014, pp. 23-25.

[62] S. B. Kotsiantis, I. D. Zaharakis, and P. E. Pintelas, "Machine learning: A review of classification and combining techniques," Artif. Intell. Rev., vol. 26, pp. 159-190, 2006.

[63] S. J. Russell, P. Norvig, J. F. Canny, J. M. Malik, D. D. Edwards, and S. J. S. Jonathan, Artificial Intelligence: A Modern Approach, vol. 13. Egnlewood Cliffs, NJ. USA: Prentice-Hall, 1995.

[64] F. Nigsch, A. Bender, B. van Buuren, J. Tissen, E. Nigsch, and J. B. O. Mitchell, "Melting point prediction employing k-nearest neighbor algorithms and genetic parameter optimization," J. Chem. Inf. Model., vol. 46, no. 6, pp. 2412-22, Jan. 2006.

[65] A. Yazdani, A. Roodaki, S. H. Rezatofighi, K. Misaghian, and S. K. Setarehdan, "Fisher linear discriminant based person identification using visual evoked potentials," in Proc. 9th Int. Conf. Signal Process., 2008, pp. 1677-1680.

[66] F. Su, L. Xia, A. Cai, and J. Ma, "Evaluation of recording factors in EEGbased personal identification: A vital step in real implementations," in Proc. IEEE Int. Conf. Syst., Man Cybern., 2010, pp. 3861-3866.

[67] G. McLachlan, Discriminant Analysis and Statistical Pattern Recognition. Hoboken, NJ, USA: Wiley, 2004.

[68] R. A. Fisher, "The use of multiple measurements in taxonomic problems," Ann. Eugen., vol. 7, no. 2, pp. 179-188, Sep. 1936.

[69] R. Palaniappan, "Electroencephalogram signals from imagined activities: A novel biometric identifier for a small population," in Proc. Intell. Data Eng. Autom. Learn., 2006, pp. 604-611.

[70] H. J. Lee, H. S. Kim, and K. S. Park, "A study on the reproducibility of biometric authentication based on electroencephalogram (EEG)," in Proc. 2013 6th Int. IEEE/EMBS Conf. Neural Eng., 2013, pp. 13-16.

[71] J. S. Kostilek, M., "EEG biometric identification: Repeatability and influence of movement-related EEG," in Proc. Int. Conf. Appl. Electron., 2012, pp. $147-150$.

[72] R. Palaniappan, "Method of identifying individuals using VEP signals and neural network," IEE Proc.-Sci., Meas. Technol., vol. 151, no. 1, pp. 16-20, 2004.

[73] R. Palaniappan, J. Gosalia, K. Revett, and A. Samraj, "PIN Generation Using Single Channel EEG Biometric," in Proc. Adv. Comput. Commun., 2011, pp. 378-385.

[74] Q. Gui and Z. Jin, "Exploring EEG-based biometrics for user identification and authentication," in Proc. Signal Process. Med. Biol. Sympo., 2014, pp. 1-6.

[75] F. Minow, "EASY CAP | EEG Recording Caps and Related Products | FMS | Falk Minow Services," FMS - Falk Minow Services, Germany, accessed on Sep. 27, 2016, [Online]. Available: www.easycap.de

[76] A. Graves, M. Liwicki, S. Fernández, R. Bertolami, H. Bunke, and J. Schmidhuber, "A novel connectionist system for unconstrained handwriting recognition,” IEEE Trans. Pattern Anal. Mach. Intell., vol. 31, no. 5, pp. 855-68, May 2009.

[77] D. Ciresan, U. Meier, and J. Schmidhuber, "Multi-column deep neural networks for image classification," in Proc. IEEE Conf. Comput. Vis. Pattern Recognit., 2012, pp. 3642-3649.

[78] A. Aizerman, E. Braverman, and L. Rozoner, "Theoretical foundations of the potential function method in pattern recognition learning," Autom. Remote Control, vol. 25, pp. 821-837, 1964.

[79] C. Cortes and V. Vapnik, "Support-vector networks," Mach. Learn., vol. 20, no. 3, pp. 273-297, Sep. 1995.

[80] H. U. Jian-feng, "Comparison of different classifiers for biometric system based on EEG signals," in Proc. 2nd Int. Conf. Inf. Technol. Comput. Sci., 2010, pp. 288-291.

[81] C. Ashby, A. Bhatia, F. Tenore, and J. Vogelstein, "Low-cost electroencephalogram (EEG) based authentication," in Proc. 5th Int. IEEE/EMBS Conf. Neural Eng., 2011, pp. 442-445.

[82] C.-W. Hsu and C.-J. Lin, "A comparison of methods for multiclass support vector machines," IEEE Trans. Neural Netw., vol. 13, no. 2, pp. 415-25, Jan. 2002.
[83] R. Palaniappan and K. V. R. Ravi, "Improving visual evoked potential feature classification for person recognition using PCA and normalization,' Pattern Recognit. Lett., vol. 27, no. 7, pp. 726-733, May 2006.

[84] S. Sun, "Multitask learning for EEG-based biometrics," in Proc. 19th Int Conf. Pattern Recognit., 2008, pp. 1-4.

[85] F. Su, L. Xia, A. Cai, Y. Wu, and J. Ma, "EEG-based personal identification: From proof-of-concept to a practical system," in Proc. 20th Int. Conf. Pattern Recognit., 2010, pp. 3728-3731.

[86] C. R. Hema and A. A. Osman, "Single trial analysis on EEG signatures to identify individuals," in Proc. 6th Int. Colloq. Signal Process. Appl., 2010, pp. 1-3.

[87] Q. Zhao, H. Peng, B. Hu, Q. Liu, and L. Liu, "Improving individual identification in security check with an EEG based biometric solution," in Brain Informatics. Berlin, Germany: Springer, 2010, pp. 145-155.

[88] B. Quintela and S. Cunha, "Biometric authentication using brain responses to visual stimuli," in Proc. 3 rd Int. Conf. Bio-Inspired Syst. Signal Process., 2010, pp. 103-112.

[89] Y. Bai, Z. Zhang, and D. Ming, "Feature selection and channel optimization for biometric identification based on visual evoked potentials," in Proc. 19th Int. Conf. Digit. Signal Process., 2014, pp. 772-776.

[90] M. Poulos, M. Rangoussi, V. Chrissikopoulos, and A. Evangelou, "Parametric person identification from the EEG using computational geometry," in Proc. 6th IEEE Int. Conf. Electron., Circuits Syst., 1999, vol. 2, pp. 1005-1008.

[91] M. Poulos, M. Rangoussi, N. Alexandris, and A. Evangelou, "Person identification from the EEG using nonlinear signal classification," Methods Inf. Med., vol. 41, no. 1, pp. 64-75, Jan. 2002.

[92] D. La Rocca, P. Campisi, and G. Scarano, "EEG biometrics for individual recognition in resting state with closed eyes," in Proc. Int. Conf. Biometrics Special Interest Group, 2012, pp. 1-12.

[93] S. Yang and F. Deravi, "On the effectiveness of EEG signals as a source of biometric information," in Proc. 3rd Int. Conf. Emerg. Security Technol., 2012, pp. 49-52.

[94] D. La Rocca, P. Campisi, and J. Sol, "EEG based user recognition using BUMP modelling," in Proc. Int. Conf. Biometrics Special Interest Group, 2013, pp. 1-12.

[95] M. V. R. Blondet and S. Laszlo, "Assessment of permanence of nonvolitional EEG brainwaves as a biometric," in Proc. IEEE Int. Conf. Identity, Security Behavior Anal., 2015, pp. 1-6.

[96] E. Maiorana, D. La Rocca, and P. Campisi, "On the permanence of EEG signals for biometric recognition," IEEE Trans. Inf. Forensics Security, vol. 11, no. 1, pp. 163-175, Jan. 2016.

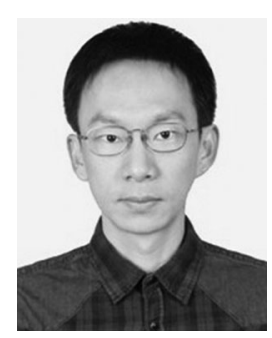

Su Yang received the B.A. degree in mechanical engineering from Changchun University of Technology, Changchun, China, in 2008, the M.Sc. degree in information technology from the University of Abertay Dundee, Dundee, U.K., in 2010, and the Ph.D. degree in electronic engineering from the University of Kent, Canterbury, U.K., in 2015

During his Ph.D. studies, he worked in the Intelligent Interactions Research Group at the School of Engineering and Digital Arts, where his research was focus on using EEG for biometric person recognition. He is currently Temple University, Philadelphia, PA, USA, as a Post-Doctoral Research Associate in the College of Engineering. His current research interests include signal processing, pattern recognition, and EEG-event detection.

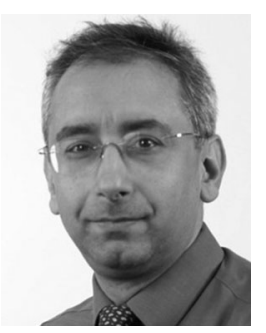

Farzin Deravi received the B.A. degree in engineering science and economics from the University of Oxford, Oxford, U.K., in 1981, the M.Sc. degree in communications engineering from Imperial College London, London, U.K., in 1982, and the Ph.D. degree in electronic engineering from the University of Wales, Cardiff, U.K., in 1988.

$\mathrm{He}$ is currently with the School of Engineering and Digital Arts, University of Kent, Canterbury, U.K., where he is a Reader in Information Engineering. His current research interests include the fields of pattern recognition and signal processing and their application in security and healthcare. 